Reprod. Nutr. Dévelop., 1984, 24 (4), 487-494.

\title{
Etude de la spermatogenèse chez le rat Wistar prépubère au cours d'une malnutrition protéino-énergétique suivie d'une réalimentation équilibrée
}

\author{
G. LAC, Nadine LAC-SCANZI \\ Avec la collaboration technique de Touria BENMOUSSAT \\ Laboratoire de Physiologie animale et de la Nutrition, \\ Institut de Biologie, Université d'Oran, \\ B.P. 76, Oran El Mnaouer, Algérie.
}

Summary. A study of spermatogenesis in prepuberal Wistar rat during protein-calorie malnutrition followed by balanced rehabilitation.

Protein-calorie malnutrition was induced by giving a $2 \%$ casein diet to a strain of postweaning male Wistar rats from 26 to 56 days of age. They were then rehabilitated by a $15 \%$ casein diet till 86 days of age. Body and testis weights, diameter of seminiferous tubules and spermatogenetic score were compared to those of controls fed with the $15 \%$ casein diet.

The unbalanced diet retarded growth by 30 days. Although rehabilitation of body and testis weights was incomplete, the spermatogenetic function equalled that of the controls at the end of the experiment. This aspect has been discussed.

\section{Introduction.}

La malnutrition exerce un effet inhibiteur incontestable sur la fonction reproductrice (Horn, 1955 ; Herbert, $1980 \mathrm{chez}$ le Rat ; Dickerson et al., $1964 \mathrm{chez}$ le Porc ; Mann, 1960 chez le Veau ; Slob et al., 1979 chez le Cobaye). Chez la Ratte, la restriction alimentaire entraîne un allongement du cycle et une baisse de fertilité si elle est adulte (Brochart et Girou, 1971) ou le non-développement des follicules si elle est impubère (Lintern-Moore et al., 1981). Le Rat mâle adulte semble moins sensible car la production de sperme n'est pas affectée par une phase de malnutrition modérée (Ghafoorunissa, 1980). Chez le mâle impubère par contre, la sensibilité de l'appareil reproducteur à la qualité du régime alimentaire est importante. En abaissant le taux de protéines de la ration à $8 \%$, Herbert (1980) obtient chez le jeune Rat une inhibition du développement des tubes séminifères, empêchant la mise en place complète de la spermatogenèse et par suite I'apparition de la puberté. C'est la totalité de l'axe hypothalamo-hypophysogonadique qui est déprimée dans ce cas comme le révèlent les dosages hormonaux (Campbell et al., 1977 ; Herbert, 1980 ; Glass et al., 1979). 
Si l'inhibition de la maturation de l'appareil reproducteur avec ce type de régime chez le jeune Rat est relativement bien documentée, la restauration de ses potentialités lors du retour à un régime équilibré n'a pratiquement jamais été considérée. Horn (1955) a seulement montré qu'elle était possible dans certains cas, mais sans en décrire les modalités.

C'est essentiellement à cet aspect que nous nous sommes intéressés dans ce travail. Nous décrivons la restauration des structures du tube séminifère et de la spermatogenèse après une période de malnutrition induite par une restriction du taux de protéines de la ration.

\section{Matériel et méthodes.}

L'étude est réalisée sur des Rats de souche Wistar provenant de l'élevage du laboratoire. Deux lots d'animaux sevrés à l'âge de 21 jours sont élevés en parallèle (fig. 1a). Le lot malnutri (M) reçoit un régime standard (15\% de caséine, 0,3\% de méthionine) de 21 à 26 jours (phase d'adaptation au « stress » du sevrage) puis

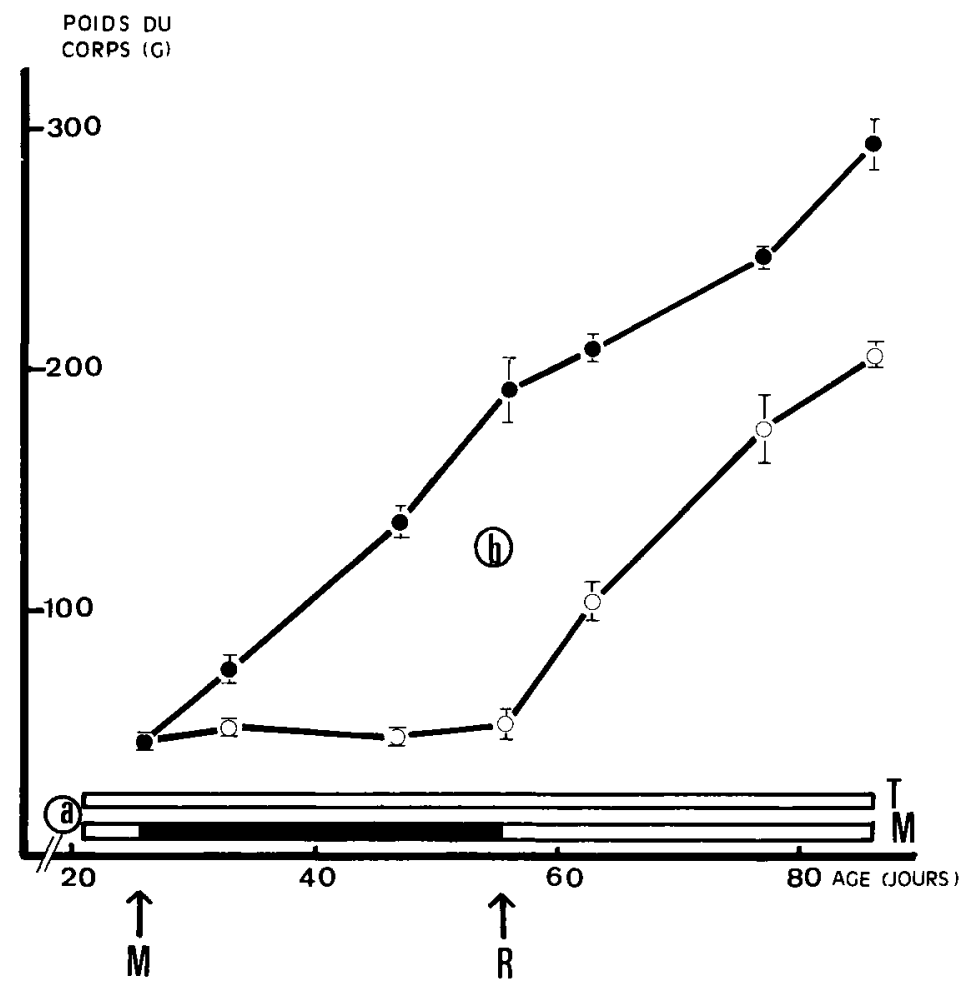

FIG. 1. - a. Protocole expérimental.

Administration du régime à $15 \%$ de caséine ; Administration du régime à $2 \%$ de caséine. $\stackrel{\uparrow}{M}$ Début de malnutrition; $\hat{R}$ Début de réhabilitation. T-Témoins ; M-Malnutris.

1 - b. Evolution du poids corporel en fonction de l'âge et du régime.

- Témoins ; -. C Malnutris. 
un régime isocalorique carencé en protéines ( $2 \%$ de caséine) de 26 à 56 jours (phase de malnutrition), puis de nouveau le régime standard jusqu'à 86 jours (phase de réhabilitation). Ce lot malnutri est comparé à un lot témoin (T) qui reçoit le régime standard pendant toute la durée du protocole. Dans tous les cas, le régime est distribué ad libitum.

Le jour 0 et les jours 7,21 et 30 de chaque phase (soit aux âges de 26, 33, $47,56,63,77$ et 86 jours) cinq animaux de chaque lot sont sacrifiés. Les différences de poids corporel et testiculaire entre témoins et malnutris sont analysées au moyen du test « $\mathrm{t} »$ de Student.

L'étude de la spermatogenèse repose sur l'observation histologique globale, la mesure du diamètre des tubes séminifères (moyenne de 100 sections orthogonales par testicule) et la détermination du score spermatogénétique. Cette dernière technique (Johnsen, 1970 ; modifiée Robert, 1980) consiste à affecter un score (de 1 à 10) au tube séminifère en fonction de l'état d'avancement de la spermatogenèse selon les critères définis dans le tableau 1. Cent tubes séminifères par testicules sont examinés et le pourcentage de chaque score représenté graphiquement.

\section{TABLEAU 1}

Détermination du score spermatogénétique.

\begin{aligned} Score & \multicolumn{1}{c}{ Etat de l'épithélium séminifère } \\ \hline 10 & Spermatogenèse complète (avec plus de 5 spermatozoïdes) \\ 9 & Spermatogenèse complète (avec moins de 5 spermatozoïdes) \\ 8 & Pas de spermatozoïdes, mais plus de 5 spermatides allongées \\ 7 & Pas de spermatozoïdes, mais moins de 5 spermatides allongées \\ 6 & Pas de spermatide allongée, mais plus de 5 spermatides rondes \\ 5 & Pas de spermatide allongée, mais moins de 5 spermatides rondes \\ 4 & Pas de spermatide, mais spermatocytes les plus avancés au stade pachytène ou diplotène \\ 3 & Pas de spermatides, mais spermatocytes les plus avancés au stade leptotène ou zygotène \\ 2 & Spermatogonies \\ 1 & Gonocytes \end{aligned}

\section{Résultats.}

La croissance pondérale corporelle (fig. 1b) est approximativement linéaire chez les témoins. Elle est nulle chez les malnutris pendant la phase de malnutrition et elle reprend immédiatement lors du retour au régime standard. Très forte au début, elle ralentit ensuite avec toutefois une tendance au rattrapage des témoins. Le déficit de $72 \%$ par rapport aux témoins à la fin de la phase de malnutrition n'est plus que de $36 \%$ à la fin de la phase de réhabilitation, ces différences étant significatives ( $\mathrm{p}<0,001$ ).

La croissance pondérale du testicule (fig. 2a) se caractérise par un infléchissement à partir de 63 jours chez les témoins ; chez les malnutris la croissance est nulle pendant les 21 premiers jours de la phase de malnutrition, elle reprend légè- 


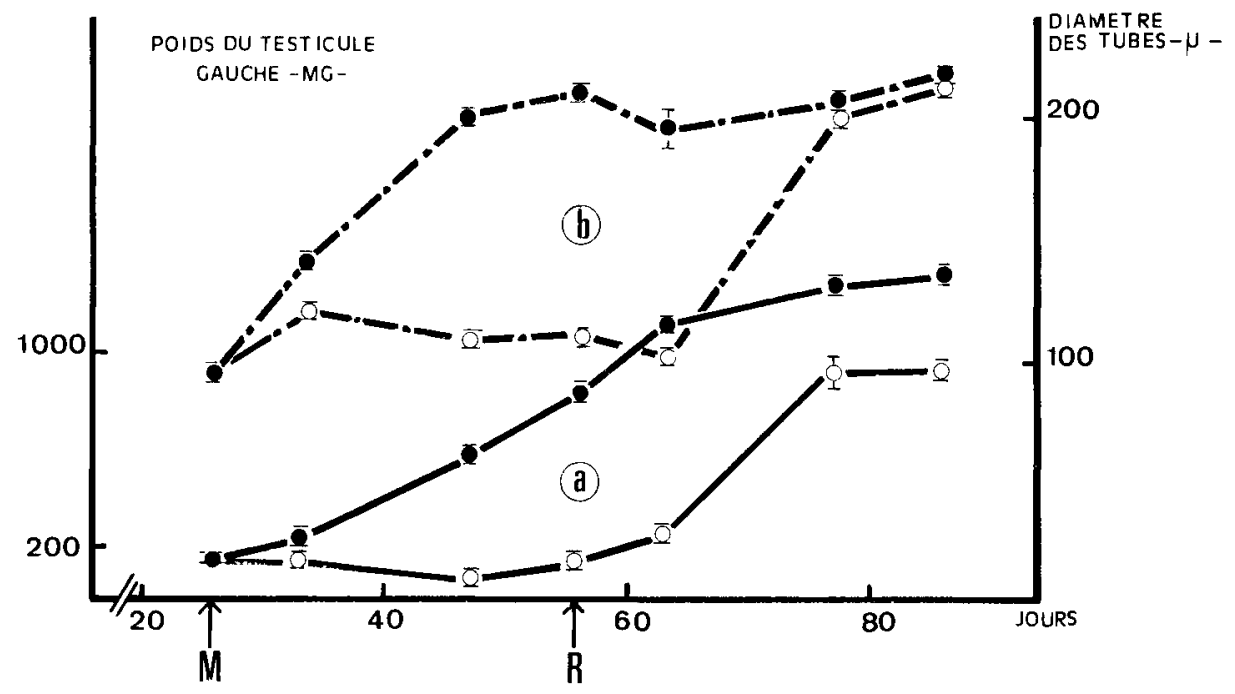

FIG. 2. - a. Evolution du poids testiculaire en fonction de l'âge et du régime. - Témoins: $\bigcirc$ Malnutris.

2 - b. Evolution du diamètre des tubes séminifères en fonction de l'âge et du régime. -.. Témoins ; - $O$ Malnutris.

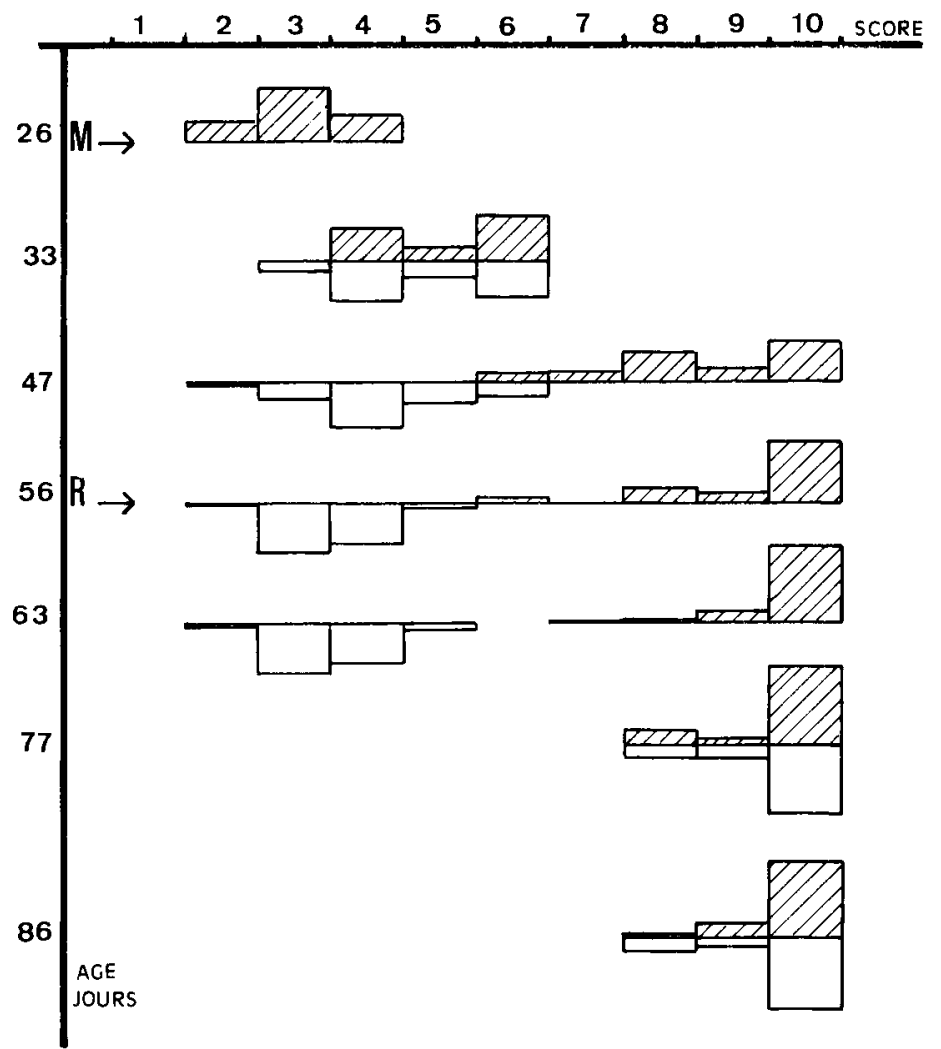

FIG. 3. - Score spermatogénétique en fonction de l'âge et du régime. एIIt Témoins ; $\square$ Malnutris. 
rement ensuite au cours de la dernière partie de cette phase entre les âges de 47 et 56 jours. Cette augmentation n'est cependant pas significative. Après 7 jours de réhabilitation, à 63 jours, le poids testiculaire a augmenté de $107 \%$ ( $p<0,01$ ) puis la croissance s'accélère fortement entre 63 et 77 jours $(+250 \%, p<0,001)$; on ne note pas ensuite d'augmentation significative entre 77 et 86 jours. En fin de phase de réhabilitation, à 86 jours, le poids testiculaire des malnutris accuse un déficit de $29 \%$ significatif par rapport aux témoins ( $p<0,001)$.

Le diamètre des tubes séminifères (fig. 2b) passe chez les témoins de $95 \mu$ à l'âge de 26 jours à $195 \mu$ à 47 jours ; sa croissance est ensuite très lente puisque le diamètre n'est que de $210 \mu$ à 86 jours. Cette valeur ne représente encore que $84 \%$ de celle d'un adulte de dix mois chez qui nous avons déterminé un diamètre moyen de tube séminifère de $250 \mu$. Chez les malnutris, le diamètre augmente de $29,7 \%$ entre 26 et 33 jours ; cette croissance est cependant inférieure à celle des témoins, ce qui se traduit par un déficit de 10,9\% par rapport aux témoins de 33 jours. Ce diamètre reste stationnaire jusqu'à 63 jours puis il augmente très fortement pour atteindre à 77 jours une valeur comparable à celle des témoins ; l'évolution est ensuite parallèle à celle des témoins.

L'étude du score spermatogénétique (fig. 3) montre qu'à 26 jours la lignée spermatogénétique ne dépasse pas le stade spermatocyte. Chez les témoins à 33 jours, plus de $80 \%$ des tubes contiennent des spermatides rondes, et à 47 jours, des tubes séminifères présentent des spermatozoïdes dans leur lumière. L'évolution se caractérise ensuite par une amélioration du rendement de la spermatogenèse (90\% de score 10 à 86 jours). Chez les malnutris, le score à 33 jours est comparable à celui des témoins de même âge. II régresse ensuite jusqu'à 56 jours pour revenir à une valeur identique à celle des animaux de 26 jours. A 63 jours, soit après 7 jours de réhabilitation, le score n'est pas amélioré, par contre à 77 jours, soit 21 jours de réhabilitation, la récupération est totale et le score est équivalent à celui des témoins de même âge.

L'observation histologique globale révèle chez les malnutris une dégénérescence du tube séminifère qui s'installe progressivement. Le nombre total de cellules par section diminue. Dès le $7^{\mathrm{e}}$ jour de malnutrition, des spermatocytes se détachent de la cellule de Sertoli pour tomber dans la lumière du tube séminifère. La lumière disparaît progressivement, envahie par un stroma dans lequel sont dispersées les cellules. La reprise du régime standard ne s'accompagne pas au début d'une progression de la spermatogenèse (cf. paragraphe précédent), mais il se produit néanmoins une réorganisation de l'épithélium séminifère. La lumière du tube réapparaît et les cellules vont se disposer contre la membrane basale ; les tubes présentent alors à 63 jours une image comparable à celle d'un témoin de 26 jours.

\section{Discussion.}

L'objectif de ce travail est d'étudier les potentialités de récupération de la fonction reproductrice après une période de malnutrition sévère installée au moment de la puberté. Dans cette optique, la première étape consiste à obtenir le 
syndrome de malnutrition protéino-énergétique (M-P-E), de façon à se placer, à partir de ce modèle expérimental, dans des conditions qui reproduisent celles observées chez l'enfant malnutri des Pays en voie de développement. Le régime utilisé, proche de celui utilisé par Okasaki et al. (1981), et déjà testé par nousmêmes (Lac et Cnockaert, 1982) satisfait à cet objectif.

$L^{\prime}$ expérimentation s'étend du $26^{\circ}$ au $86^{\circ}$ jour d'âge, soit pendant la phase de croissance linéaire rapide du Rat (Gosse et al., 1970), au cours de laquelle a lieu la puberté. L'évolution du poids testiculaire est biphasique (Desjardins et al., 1968 ; Brown-Grant et al., 1975) ; la croissance est en effet fortement ralentie à partir de 63 jours chez les témoins. L'administration du régime à $2 \%$ de caséine provoque un arrêt immédiat des croissances corporelle et testiculaire. Toutefois, au cours des 10 derniers jours de la phase de malnutrition, il se produit une légère augmentation du poids testiculaire, phénomène déjà noté par Herbert (1980). Lors du retour au régime standard après la phase de malnutrition, le poids testiculaire ne présente pas de croissance compensatrice immédiate comme c'était le cas pour le poids corporel ; la reprise ne devient nette qu'au bout d'une semaine de réhabilitation.

L'évolution du diamètre des tubes séminifères reproduit en l'accentuant celle du poids testiculaire. A noter cependant chez les témoins que le palier qui se produit à 63 jours pour le poids testiculaire se produit plus précocement (entre 50 et 55 jours) pour le diamètre des tubes séminifères, et chez les malnutris, que la récupération est totale dès l'âge de 77 jours, à la différence des poids corporel et testiculaire qui conservent un déficit par rapport au témoin jusqu'à la fin de l'expérimentation.

L'examen de l'épithélium séminifère par la méthode du score spermatogénétique montre que les spermatozoïdes sont présents dans la lumière du tube séminifère à l'âge de 47 jours, en accord avec Clermont et al. (1959), dans cette espèce. Environ $50 \%$ des tubes sont affectés du score 10, valeur voisine de celle fournie par Brown-Grant et al. (1975) (48\% à 50 jours), dans la même souche. Le rendement de la spermatogenèse s'accroît ensuite jusqu'à $90 \%$ à 86 jours ; ceci serait dû selon Hochereau de Reviers (1970) à une augmentation du nombre de lignées spermatogénétiques entrant en division, et rendrait compte de l'augmentation de poids testiculaire post-pubertaire.

Chez les malnutris, l'évolution du score spermatogénétique est identique à celle des témoins jusqu'à 33 jours. II y a donc concordance entre l'évolution de ce paramètre et celle du diamètre des tubes séminifères. L'évolution ultérieure se caractérise par une régression, déjà nette à 47 jours, qui s'amplifie par la suite, ramenant le score des malnutris de 56 et 63 jours au niveau de celui des témoins de 26 jours. Ceci est en accord avec l'observation de cellules en cours de dégénérescence dans la lumière du tube notées également par Herbert (1980). Le fait que les cellules desquamées dans la lumière correspondent fréquemment à des images de spermatocytes alors que le niveau du score 4 ne régresse pas, laisse penser que le fonctionnement de la lignée germinale n'est pas aboli, mais qu'elle est incapable de dépasser le stade spermatocyte, voire quelques spermatides rondes (st. 5).

La reprise du régime standard n'entraîne pas d'amélioration du score au 
cours des 7 premiers jours, mais simplement une réorganisation de l'épithélium séminifère avec reconstitution de la lumière, ce qui aboutit à 63 jours à une image identique à celle des témoins de 26 jours. Par contre, entre 63 et 77 jours, la reprise est explosive, et à 77 jours les malnutris présentent un score comparable à celui des témoins de même âge. L'arrêt de la malnutrition entraîne donc la levée du blocage signalé plus haut sur la spermatogenèse et la poursuite de la maturation jusqu'au stade spermatozoïde. II y a pour ce paramètre un rattrapage chronologique intégral alors qu'il n'est que partiel pour le poids de la glande et le poids du corps. Ceci permet de supposer que pour la fonction reproductrice, après une période de malnutrition sévère, le rattrapage fonctionnel précède le retour à la valeur témoin des paramètres pondéraux.

Reçu en janvier 1984.

Accepté en mars 1984.

Remerciements. - A Monsieur le Professeur Delost P. et Monsieur le Docteur Robert A. pour avoir bien voulu examiner le manuscrit.

\section{Références}

BROCHART M., GIROU R., 1971. Réduction brutale ou progressive du niveau alimentaire et fertilité chez la Ratte. Influence du stade du cycle œestral auquel intervient la réduction. $C$. $R$. Soc. Biol., 165, 883-886.

BROWN-GRANT K., FINK G., GREIG F., MURRAY M. A. F., 1975. Altered sexual development in male rat after oestrogen administration during the neonatal period. J. Reprod. Fert., 44, 25-42.

CAMPBELL G. A., KURCZ M., MARSHALL S., MEITES J., 1977. Effects of starvation in rats on serum levels of follicle stimulating hormone, luteinizing hormone, thyrotropin hormone, growth hormone and prolactin ; response to $\mathrm{LH}$-releasing hormone and thyrotropin hormone. Endocrinology, 100, 580-587.

CLERMONT Y., LEBLOND C. P., MESSIER B., 1959. Durée du cycle de l'épithélium séminal du Rat. Arch. Anat. microscop. Morphol. exper., 48, 37-56.

DESJARDINS C., MACMILLAN K. L., HAFS H. D., 1968. Reproductive organ DNA and RNA of male and female rats from birth to 100 days of age. Anat. Rec., 161, 17-22.

DICKERSON J. W., GRESHAM G. A., Mc CANCE R. A., 1964. The effect of undernutrition and rehabilitation on the development of the reproductive organs : pigs., J. Endocr., 29, 111118.

GHAFOORUNISSA, 1980. Undernutrition and fertility of male rat. J. Reprod. Fert., 59, 317-320.

GLASS A., MELLIT R., VIGERSKI R. A., SWERDLOFF A. S., 1979. Hypoandrogenism and abnormal regulation of gonadotropin in rats fed a low protein diet. Endocrinology, 104, 438-442.

GOSSE C., HUARD M., DECHAMBRE R. P., CARDI P., 1970. Influence du rationnement et de la densité de population sur l'évolution pondérale et la consommation de nourriture du Rat Wistar mâle. Exp. anim., 3, 245-262.

HERBERT C. D., 1980. Growth patterns and hormonal profile of male rat with protein-calorie malnutrition. Anat. Rec., 197, 339-354.

HOCHEREAU-de REVIERS M. T., 1970. Variation in the stock of testicular stem cells in the yield of spermatogonial divisions in the ram and bull testis. Andrologia, 8, 137-146.

HORN E. H., 1955. Nutritional and hormonal influences upon reproductive maturation, organ weights and histochemistry of the immature male rat. Endocrinology, 57, 399-408. 
JOHNSEN S. G., 1970. Testicular biopsy count : a method for registration of spermatogenesis in human testis : normal values and results in 335 hypogonadial males. Hormones, 1, 1-5.

LAC G., CNOCKAERT J. C., 1982. Evolution de la consommation minimale d'oxygène chez le jeune Rat au cours d'une malnutrition protéino-énergétique suivie d'une réalimentátion équilibrée. Arch. int. Physiol. Bioch., 90, 301-308.

LINTERN-MOORE S., EVERITT A. V., MARIANA J. C., MAULÉON P., 1981. The effect of restricted food intake and refeeding on the ovarian follicle population of the prepuberal Wistar rat. Reprod. Nutr. Dévelop., 21, 611-622.

MANN T., 1960. Effect of nutrition on androgenic activity and spermatogenesis in mammals. Proc. Nutr. Soc., 19, 15-18.

OKASAKI S., MATSUEDA S., OHNAKA M., NIIYAMA Y., 1981. Effect of various periods of protein restriction immediately after weaning on subsequent catch-up growth in rat. Nutr. Rep. int., 23, 471-485.

ROBERT A., 1980. L'établissement de la spermatogenèse chez le Cobaye à la puberté. C. R. Soc. Biol., 174, 262-267.

SLOB A. K., VREEBURG J. T. M., VAN DER WERFF TEN BOSCH J. J., 1979. Body growth, puberty and undernutrition in the male guinea-pig. Br. J. Nutr., 41, 231-237. 